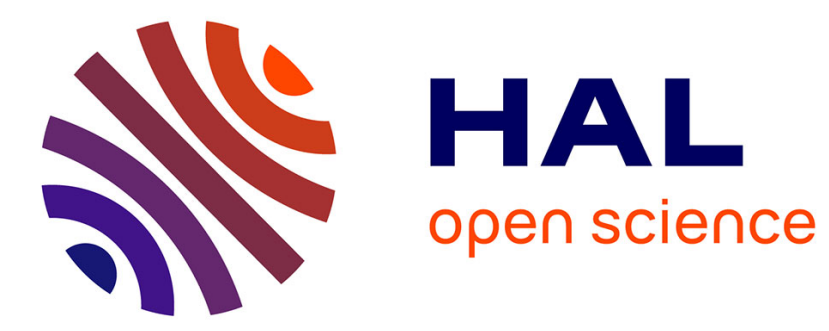

\title{
Appropriateness of blood culture testing parameters in routine practice. Results from a cross-sectional study
}

V. Vitrat-Hincky, P. François, J. Labarère, C. Recule, J. P. Stahl, P. Pavese

\section{To cite this version:}

V. Vitrat-Hincky, P. François, J. Labarère, C. Recule, J. P. Stahl, et al.. Appropriateness of blood culture testing parameters in routine practice. Results from a cross-sectional study. European Journal of Clinical Microbiology and Infectious Diseases, 2010, 30 (4), pp.533-539. 10.1007/s10096-010-11158. hal-00642418

\section{HAL Id: hal-00642418 \\ https://hal.science/hal-00642418}

Submitted on 18 Nov 2011

HAL is a multi-disciplinary open access archive for the deposit and dissemination of scientific research documents, whether they are published or not. The documents may come from teaching and research institutions in France or abroad, or from public or private research centers.
L'archive ouverte pluridisciplinaire HAL, est destinée au dépôt et à la diffusion de documents scientifiques de niveau recherche, publiés ou non, émanant des établissements d'enseignement et de recherche français ou étrangers, des laboratoires publics ou privés. 
Appropriateness of Blood Culture Testing Parameters in Routine Practice. Results from a Cross-Sectional Study.

V Vitrat-Hincky ${ }^{1}$, P François $^{2,3}$, J Labarère $^{2,3}$, C Recule $^{4}$, JP Stahl $^{1}$, P Pavese $^{1}$

\section{Author affiliations:}

${ }^{1}$ Department of Infectious diseases. Grenoble University Hospital, Grenoble, France.

${ }^{2}$ Quality of Care Unit. Grenoble University Hospital, Grenoble, France.

${ }^{3}$ TIMC, UMR 5525 CNRS Université Joseph Fourier, Grenoble France

${ }^{4}$ Laboratory of Bacteriology. Grenoble University Hospital, Grenoble, France.

Corresponding author: Virginie Vitrat-Hincky

Clinique de Maladies Infectieuses et Tropicales

CHU de Grenoble- $3^{\text {ème }} \mathrm{F}$

38043 Grenoble Cedex 9

France

VHinckyvitrat@chu-grenoble.fr

Phone: +33476765291/ +33619923327

Fax: +33476765838

Running head: Blood culture practice 


\begin{abstract}
Purpose. We aimed to assess the appropriateness of routine blood culture testing parameters and antimicrobial therapy for patients with suspected bloodstream infection.

Methods. We conducted a cross-sectional study of blood cultures registered in the microbiological laboratory at a university-affiliated hospital from 4 to 15 June 2007. Using a structured implicit chart review, two infectious disease specialists assessed the appropriateness of the testing parameters and antimicrobial therapy.
\end{abstract}

Results. Overall, 2696 blood culture bottles were collected from 260 patients during their stay, including 955 bottles that were evaluated during the study period. The indication of fungal and bacterial blood cultures was rated as appropriate for $75 \%$ (95\% confidence interval $[\mathrm{CI}], 65-83$ ) and $91 \%$ (95\% CI, 87-95) of patients. Only 45\% (95\% CI, 39-52) of patients had an adequate number of blood cultures (i.e., two to four). An optimal volume of blood (i.e., $\geq 10 \mathrm{~mL}$ ) was inoculated in $13 \%$ (95\% CI, 11-15) of adult bottles. Empirical antimicrobial therapy was appropriate for $60 \%$ (95\% CI, 43-76) of patients with positive blood cultures.

Conclusions. There is room for improvement regarding routine blood culture testing parameters and antimicrobial therapy. The effectiveness of multifaceted interventions in altering the appropriateness of blood culture parameters deserves further research.

Keywords: Bacteremia; Blood specimen collection; Blood; Microbiology; Cross-sectional studies; Hospital 


\section{INTRODUCTION}

Early diagnosis and prompt administration of appropriate antimicrobial therapy are essential for patients with bloodstream infections (BSI), because of the morbidity and mortality associated with this condition $[1,2]$. Thus in febrile patients, blood cultures are frequently used to identify causative pathogens in the hospital setting. Yet, only $5-15 \%$ of blood cultures yield a clinically significant pathogen in routine practice $[3,4]$. Even when positive, blood cultures may be difficult to interpret and false-positive cases result in increased costs and inappropriate use of antibiotics [5-7]. Furthermore, the value of blood culture results has been questioned for certain disorders such as acute uncomplicated pyelonephritis in women or community-acquired pneumonia because they seldom provide additional information that leads to treatment alteration [8].

Several factors influencing blood culture yield have been consistently reported including the volume of blood drawn into the culture bottle, number of blood culture sets, timing and intervals of blood cultures, and sites for obtaining blood samples [9-14]. Despite recommendations advocating optimal testing parameters for blood cultures [9-14], anecdotal and survey evidence suggests that clinical practices pertaining to this issue are often inappropriate $[15,16]$.

The aim of this study was to assess the appropriateness of the indication and testing parameters for blood cultures obtained from hospitalized patients. We also analyzed empirical antimicrobial therapy and adjustment of antimicrobial treatment after the results of blood cultures were available. 


\section{MATERIALS AND METHODS}

Study design

We conducted a cross-sectional study of blood cultures that were submitted to the microbiological laboratory at a 1,500-bed university-affiliated hospital in France. In this observational study, blood cultures were ordered at the discretion of attending physicians and there was no recommendation policy regarding the number of blood cultures at our institution. However, local guidelines for skin preparation, sites, volume, and collecting procedure were available on the hospital's intranet Web site.

Patients

Microbiologists prospectively identified consecutive patients from whom blood cultures were obtained from 4 to 15 June 2007. All medical, surgical, pediatric, emergency department, and intensive care unit patients were eligible.

Data collection

A physician abstracted information on age, gender, department, comorbid conditions, intravascular access devices, clinical signs and symptoms of bacteremia or fungemia, relevant laboratory and x-ray findings, and antimicrobial therapy. The patients were followed up until discharge from the hospital and the length of stay and discharge diagnoses were also recorded.

Laboratory procedures and definitions

A microbiologist prospectively collected information on the site, timing, and volume of blood cultures that were submitted during the study period. In accordance with previous studies [10], a blood culture was defined as a sample of blood obtained from a single puncture site, whether that blood was inoculated into one or multiple bottles. With the exception of pediatric patients, the routine approach at our institution consisted of inoculating one aerobic and one 
anaerobic bottle per set. Taking fungal blood cultures was not systematic and required a special prescription from the attending physician.

The samples were cultured using the BACTEC 9000 (Becton Dickinson, USA) system with BACTEC Plus Aerobic/F resin, BD BACTEC Lytic 10 Anaerobic/F, BACTEC PEDS Plus/F, and BACTEC Mycosis IC/F bottles. In cases of identification of pathogen bacteria such as Staphylococcus aureus, Streptococcus spp. or Enterobacteriaceae, the blood cultures were classified as true positive. In cases of potentially contaminant bacteria such as coagulase-negative Staphylococcus, the blood cultures were classified as true positive if more than one sample was positive, clinical or biological signs of infection were present, and the patient was immunosuppressed or had an intravascular catheter.

Appropriateness measures

Using a structured implicit chart review, two infectious disease specialists independently assessed the appropriateness of the blood culture testing parameters. Structured implicit chart review relies on expert judgment of the quality of care for an individual patient case with the key aspects of care and data sources to be reviewed specified in advance. More specifically, the parameters assessed for appropriateness in this study included the indication for blood culture, the site for obtaining the blood sample, empirical antimicrobial therapy, and antimicrobial treatment adjustment after the results of blood cultures were available. For all measures, appropriateness was rated on a 4-point scale (i.e., very appropriate, fairly appropriate, fairly inappropriate, and very inappropriate). The infectious disease specialists were instructed to assess the appropriateness of the blood culture and empirical antimicrobial therapy, regardless of blood culture yield and patient outcomes. Their assessment of empirical antimicrobial therapy was based on current guidelines considering the site and type of initially suspected infection and the patient's comorbid conditions. The initial diagnosis was approached using clinical, laboratory, and x-ray findings that were available when the first blood culture set was collected. The appropriateness of the initial empirical 
antimicrobial therapy was not secondarily re-evaluated based on the blood culture yield. Inter-rater reliability was not formally assessed in this study.

Additionally, we evaluated the total number of blood cultures and the interval between consecutive blood cultures during the hospital stay for each patient. We also measured the volume of blood inoculated per bottle during the study period. Based on current guidelines $[9,11,13]$, we considered that the appropriate number of blood cultures ranged from two to four, two consecutive blood samples had to be obtained within a 24-h time frame, and the appropriate volume of blood per bottle was $10 \mathrm{~mL}$ or more for adult patients and $3 \mathrm{~mL}$ or more for pediatric patients.

Statistical analyses

Baseline characteristics were reported as medians and interquartile ranges (IQR; i.e., $25^{\text {th }}$ and $75^{\text {th }}$ percentiles) for continuous variables and percentages for categorical variables. We compared baseline characteristics between study groups using the $\chi^{2}$ test or the Fisher exact test where appropriate for categorical variables and the Wilcoxon test for continuous variables. Exact 95\% confidence intervals were computed from the binomial distribution using Stata 9 (Stata Corporation, College Station, TX, USA). 


\section{RESULTS}

Overall, 2696 blood culture bottles were collected from 260 ( 224 adult and 36 pediatric) patients during their stay, including 955 bottles that were evaluated during the study period. The median age for all patients was 56 years (IQR, 36-75), and 159 patients (61\%) were male (Table 1). Hypothermia $\left(\leq 36^{\circ} \mathrm{C}\right)$ or fever $\left(\geq 38.3^{\circ} \mathrm{C}\right)$ was present in 152 patients $(68 \%)$. Overall, 32 patients had pathogens isolated from blood cultures, five patients had contaminated blood cultures, and one patient had a true-positive and a contaminated blood culture. Of the 33 patients with pathogens isolated from blood cultures, 32 had monomicrobial BSI and one had polymicrobial BSI. The most commonly recovered microorganisms were Enterobacteriaceae, Staphylococcus aureus, and coagulase-negative staphylococci (Table 2). True-positive cases were detected with the first (30 patients) or second (three patients) blood culture sets. The $7^{\text {th }}$ and $11^{\text {th }}$ sets were positive only for two patients but they were considered contaminated (Figure 1). No fungal microorganisms were isolated. The most common discharge diagnoses were respiratory tract infection and urinary tract infection (Table 1).

The two infectious disease specialists rated the indication as very or fairly appropriate for $75 \%$ and $91 \%$ of fungal and bacterial blood cultures, respectively (Table 3). Only 118 patients $(45 \%)$ had an adequate number of blood cultures (i.e., two to four), while a single blood sample was cultured for 72 patients (28\%) and five or more blood samples were cultured for 70 patients (27\%). Patients with single blood cultures were younger (median age, 49 versus 59 years, $P=.04$ ), were less likely to have intravenous access devices ( $11 \%$ versus $27 \%, P=.006)$, and had a shorter length of stay (median, 5 versus 15 days, $P<.001$ ) than patients with two or more blood cultures. They also showed a nonsignificant trend toward a lower rate of positive blood cultures ( $8 \%$ versus $17 \%, P=.06)$.

The site the blood sample was drawn was either unspecified or inappropriate in 32 patients (12\%). Inappropriate blood culture sites were related to samples drawn from an indwelling vascular 
catheter only without a peripheral blood sample puncture. An optimal volume of blood was inoculated for only $13 \%$ of the bottles obtained from adult patients.

Regardless of the yield of blood cultures, the two infectious disease specialists rated the empirical antimicrobial therapy as very or fairly appropriate for 23 of $38(60 \%)$ patients with positive blood cultures (Table 3). They rated as very or fairly appropriate the antimicrobial therapy that was ordered after the results of blood cultures were available for 27 patients (71\%). The reasons for inappropriate adjusted antimicrobial therapy included the use of antibiotic agents that were not recommended for the treatment of BSI in six patients, the use of antibiotic agents that were inactive against the isolated microorganisms in vitro in three patients, and the use of broadspectrum antibiotic agents where antibiotic agents with a narrower spectrum would have been appropriate in two patients. 


\section{DISCUSSION}

This cross-sectional study highlights the room for improvement regarding routine blood culture testing parameters and antimicrobial therapy for patients with suspected BSI. These findings are of clinical significance because of the potential implications of both unrecognized bacteremia episodes and inappropriate antimicrobial treatment.

Despite a proven relationship between the number of blood cultures and detection rates [14], adequate numbers of blood cultures were ordered in only $45 \%$ of patients in this study. Guidelines recommend that two to three blood cultures be obtained over a 24-h period in order to maximize the detection of BSI [9, 11-13]. However, more recent studies suggest that two blood cultures detect approximately $80 \%-90 \%$ of BSI, and as many as four blood cultures may be needed over a $24-\mathrm{h}$ period to achieve a detection rate of $99 \%$ or more $[10,17]$. Due to a detection rate as low as $65 \%$ [10], single blood cultures, which were observed in $28 \%$ of the patients in our study, are inappropriate in routine practice. Our study estimates of positive blood cultures were consistent with the rates of positive blood cultures recorded for patients with single (244/5216 [4\%]) and two or more $(1000 / 5611[18 \%])$ blood cultures in our microbiological laboratory in $2007(P<.001)$. However, it should be noted that patients with single blood cultures were in less severe condition than those who underwent two or more blood cultures.

Moreover, an excessive number of blood cultures (i.e., five or more) was found in $27 \%$ of patients. The value of repeating blood cultures has been questioned because of the associated costs, unnecessary laboratory workload, and risk for nosocomial anemia [12]. A previous investigation showed that the most common reasons for clinicians repeating blood cultures include a new septic episode, persistent fever, and leukocytosis [18]. Importantly, evidence supporting the effectiveness of guidelines for requesting an appropriate number of blood cultures is currently limited [19]. 
Another striking finding of our study was the $87 \%$ rate of bottles containing an insufficient volume of blood for adult patients, with the potential for a substantial number of BSI going undiagnosed. Indeed, there is strong evidence supporting a direct relationship between the volume of blood and the yield of the culture $[15,20]$. In a retrospective study of blood cultures from infants and children, Gonsalves et al. evidenced a higher contamination rate for low-volume blood samples [21]. Some authors speculate that few clinicians and nurses are aware of the influence of the volume of blood on the sensitivity of blood cultures [15]. Insufficient volumes of blood may also result from some patients' clinical condition. Indeed, Bouza et al. reported that lower volumes of blood were observed among severely ill patients who are more likely to have BSI and for whom sample collection is also more difficult [22].

Because the indications for obtaining blood samples are broad and ill defined [11], the finding that only $75 \%-91 \%$ of fungal and bacterial blood cultures were appropriate in our study should be interpreted with caution. Nevertheless, previous research has demonstrated that physicians may overestimate the likelihood of bacteremia for most patients [23], leading to potentially unnecessary blood cultures. Several clinical decision rules have been derived in order to help the physician identify patients from whom blood samples should be obtained and cultured [24, 25]. Because of a lack of both sensitivity and external validity, these rules have limited value and have not come into widespread use $[4,13,26]$.

Concerns may exist regarding the relevance of two criteria that were assessed in our study. First, obtaining a blood sample from an intravascular catheter is a highly debated issue [27]. In our study, most blood culture collection complied with guidelines recommending that at least one blood sample, if not all, be obtained by venipuncture $[9,11]$. Second, several studies have raised the question of routine inoculation of anaerobic bottles and some guidelines recommend inoculating two bottles per set and incubating both of them aerobically with only selective use of anaerobic bottles [11]. Consistent with the findings reported by Grohs et al. [28], 
Enterobacteriaceae was recovered from anaerobic bottles in the absence of any growth in the aerobic bottle for two patients in our study. Based on this finding, the current approach of inoculating one aerobic and one anaerobic bottle per set with the selective use of fungal bottles seems appropriate in our hospital.

Prompt administration of appropriate antibiotic treatment is associated with improved survival and clinical outcomes, shorter length of stay, and decreased costs for patients with BSI [29-31]. The appropriateness of empirical antimicrobial treatment (60\%; 95\% CI, 43-76) was assessed regardless of the yield of blood cultures in our study and may not compare with estimates reported previously $[29,30]$. Despite the limited sample size for testing this criterion, the adequacy of antimicrobial therapy after the microbiological results were available (71\%; 95\% CI, 54\%-85\%) was comparable to that observed in previous studies [29, 30, 32, 33].

The potential limitations of our study deserve consideration. First, we did not investigate whether blood cultures were obtained from all patients with suspected BSI during the study period. Actually, this was not within the scope of this laboratory-based study, which focused on the appropriateness of blood cultures that were submitted to the microbiological laboratory.

Second, structured implicit chart review may be unreliable when evaluating the appropriateness of specific processes of care [34]. Although we did not formally assess inter-rater reliability in this study, we reported $\kappa$ coefficients ranging from 0.86 to 0.97 in a previous study on the appropriateness of urine cultures [35].

Third, six out of 85 positive blood cultures represented contaminants in our study (7\%, 95\% CI, 3-15). This point estimate was low compared with previous studies and our results may not extend to other settings.

In summary, this cross-sectional study questions the appropriateness of routine blood culture testing parameters and antimicrobial therapy for patients with suspected BSI. Further 
research is needed to determine the effectiveness of multifaceted interventions in altering the appropriateness of blood culture parameters. 


\section{ACKNOWLEDGMENT}

Ms. Linda Northrup from English Solutions, Voiron, France, provided assistance in editing the manuscript.

This study was supported by a grant from the Direction de la Recherche Clinique at Grenoble University Hospital. 


\section{REFERENCES}

1. Bearman GM, Wenzel RP (2005) Bacteremias: a leading cause of death. Arch Med Res $36: 646-659$.

2. Wisplinghoff H, Bischoff T, Tallent SM, Seifert H, Wenzel RP, Edmond MB (2004) Nosocomial bloodstream infections in US hospitals: analysis of 24,179 cases from a prospective nationwide surveillance study. Clin Infect Dis 39:309-317.

3. Bates DW, Cook EF Goldman L, Lee TH (1990) Predicting bacteremia in hospitalized patients. A prospectively validated model. Ann Intern Med 113:495-500.

4. Paul M, Andreassen S, Nielsen AD, Tacconelli E, Almanasreh N, Fraser A, Yahav D, Ram R, Leibovici L (2006) Prediction of bacteremia using TREAT, a computerized decisionsupport system. Clin Infect Dis 42:1274-1282.

5. Bates DW, Goldman L, Lee TH (1991) Contaminant blood cultures and resource utilization. The true consequences of false-positive results. JAMA 265:365-369.

6. Hall KK, Lyman JA (2006) Updated review of blood culture contamination. Clin Microbiol Rev 19:788-802.

7. Weinstein MP (2003) Blood culture contamination: persisting problems and partial progress. J Clin Microbiol 41:2275-2278.

8. Peters RP, Van Agtmael MA, Danner SA, Savelkoul PH, Vandenbroucke-Grauls CM (2004) New developments in the diagnosis of bloodstream infections. Lancet Infect Dis 4:751-760.

9. Chandrasekar PH, Brown WJ (1994) Clinical issues of blood cultures. Arch Intern Med 154:841-849.

10. Cockerill FR, $3^{\text {rd }}$, Wilson JW, Vetter EA, Goodman KM, Torgerson CA, Harmsen WS, Schleck CD, Ilstrup DM, Washington JA, $2^{\text {nd }}$, Wilson WR (2004) Optimal testing parameters for blood cultures. Clin Infect Dis 38:1724-1730. 
11. Mylotte JM, Tayara A (2000) Blood cultures: clinical aspects and controversies. Eur J Clin Microbiol Infect Dis 19:157-163.

12. Reimer LG, Wilson ML, Weinstein MP (1997) Update on detection of bacteremia and fungemia. Clin Microbiol Rev 10:444-465.

13. Shafazand S, Weinacker AB (2002) Blood cultures in the critical care unit: improving utilization and yield. Chest 122:1727-1736.

14. Weinstein MP (1996) Current blood culture methods and systems: clinical concepts, technology, and interpretation of results. Clin Infect Dis 23:40-46.

15. Mermel LA, Maki DG (1993) Detection of bacteremia in adults: consequences of culturing an inadequate volume of blood. Ann Intern Med 119:270-272.

16. Washington JA (1992) An international multicenter study of blood culture practices. The International Collaborative Blood Culture Study Group. Eur J Clin Microbiol Infect Dis 11:1115-1128.

17. Lee A, Mirrett S, Reller LB, Weinstein MP (2007) Detection of bloodstream infections in adults: how many blood cultures are needed? J Clin Microbiol 45:3546-3548.

18. Tabriz MS, Riederer K, Baran J, Jr, Khatib R (2004) Repeating blood cultures during hospital stay: practice pattern at a teaching hospital and a proposal for guidelines. Clin Microbiol Infect 10:624-627.

19. Gross PA, Van Antwerpen CL, Hess WA, Reilly KA (1988) Use and abuse of blood cultures: program to limit use. Am J Infect Control 16:114-117.

20. Weinstein MP, Mirrett S, Wilson ML, Reimer LG, Reller LB (1994) Controlled evaluation of 5 versus 10 milliliters of blood cultured in aerobic BacT/Alert blood culture bottles. $\mathrm{J}$ Clin Microbiol 32:2103-2106.

21. Gonsalves WI, Cornish N, Moore M, Chen A, Varman M (2009) Effects of volume and site of blood draw on blood culture results. J Clin Microbiol 47:3482-3485. 
22. Bouza E, Sousa D, Rodriguez-Creixems M, Lechuz JG, Munoz P (2007) Is the volume of blood cultured still a significant factor in the diagnosis of bloodstream infections? J Clin Microbiol 45:2765-2769.

23. Poses RM, Anthony M (1991) Availability, wishful thinking, and physicians' diagnostic judgments for patients with suspected bacteremia. Med Decis Making 11:159-168.

24. Jaimes F, Arango C, Ruiz G, Cuervo J, Botero J, Velez G, Upegui N, Machado F (2004) Predicting bacteremia at the bedside. Clin Infect Dis 38:357-362.

25. Leibovici L, Greenshtain S, Cohen O, Mor F, Wysenbeek AJ (1991) Bacteremia in febrile patients. A clinical model for diagnosis. Arch Intern Med 151:1801-1806.

26. Lautenbach E, Localio R, Nachamkin I (2004) Clinicians required very high sensitivity of a bacteremia prediction rule. J Clin Epidemiol 57:1104-1106.

27. Falagas ME, Ierodiakonou V, Alexiou VG (2009) Clinical practice of obtaining blood cultures from patients with a central venous catheter in place: an international survey. Clin Microbiol Infect 15:683-686.

28. Grohs P, Mainardi JL, Podglajen I, Hanras X, Eckert C, Buu-Hoi A, Varon E, Gutmann L (2007) Relevance of routine use of the anaerobic blood culture bottle. J Clin Microbiol 45:2711-2715.

29. Berild D, Mohseni A, Diep LM, Jensenius M, Ringertz SH (2006) Adjustment of antibiotic treatment according to the results of blood cultures leads to decreased antibiotic use and costs. J Antimicrob Chemother 57:326-230.

30. Bouza E, Sousa D, Munoz P, Rodriguez-Creixems M, Fron C, Lechuz JG (2004) Bloodstream infections: a trial of the impact of different methods of reporting positive blood culture results. Clin Infect Dis 39:1161-1169.

31. Fraser A, Paul M, Almanasreh N, Tacconelli E, Frank U, Cauda R, Borok S, Cohen M, Andreassen S, Nielsen AD, Leibovici L (2006) Benefit of appropriate empirical antibiotic treatment: thirty-day mortality and duration of hospital stay. Am J Med 119:970-976. 
32. Arbo MD, Snydman DR (1994) Influence of blood culture results on antibiotic choice in the treatment of bacteremia. Arch Intern Med 154:2641-2645.

33. Gross PA, Barrett Tl, Dellinger EP, Krause PJ, Martone WJ, Mcgowan JE, Jr, Sweet RL, Wenzel RP (1994) Quality standard for the treatment of bacteremia. The Infectious Diseases Society of America. Infect Control Hosp Epidemiol 15:189-192.

34. Hayward RA, Mcmahon LF, Jr, Bernard AM (1993) Evaluating the care of general medicine inpatients: how good is implicit review? Ann Intern Med 118:550-556.

35. Pavese P, Saurel N, Labarère J, Decouchon C, Vittoz JP, Foroni L, Maurin M, Francois P (2009) Does an educational session with an infectious diseases physician reduce the use of inappropriate antibiotic therapy for inpatients with positive urine culture results? A controlled before-and-after study. Infect Control Hosp Epidemiol 30:596-599. 
Table 1. Patient characteristics $(n=260)$.

Characteristics $^{1}$

Men, no. (\%)

$\underline{159} \underline{(61)}$

$\underline{\text { Age, median (IQR), } y}$

$\underline{56} \quad \underline{(36-75)}$

Department, no. (\%)

$\underline{\text { Medical }}$

$\underline{156} \underline{(60)}$

$\underline{\text { Surgical }}$

$\underline{22} \quad \underline{(8.4)}$

$\underline{\text { Pediatrics }}$

$\underline{36} \quad \underline{(14)}$

Emergency

$\underline{12} \underline{(4.6)}$

$\underline{\text { Intensive care unit }}$

$\underline{34} \underline{(13)}$

$\underline{\text { Temperature } \leq 36^{\circ} \mathrm{C} \text { or } \geq 38.3^{\circ} \mathrm{C}}$

$\underline{152 / 222} \underline{(68)}$

$\underline{\text { Immune suppression, no. }(\%)^{2}}$

$\underline{56} \quad \underline{(21)}$

Intravenous access device, no. (\%)

$\underline{57 / 251} \underline{(23)}$

Blood culture results, no. $(\%)^{3}$

$\underline{\text { Pathogens }}$

$\underline{33} \underline{(13)}$

$\underline{\text { Contaminated }}$

$\underline{6} \quad \underline{(2)}$

$\underline{\text { Negative }}$

$\underline{222} \underline{(85)}$

Empirical antimicrobial therapy, no. (\%)

$\underline{162} \underline{(62)}$

Discharge diagnosis, no. (\%)

$\underline{\text { Respiratory tract infection }}$

$\underline{56} \quad \underline{(22)}$

$\underline{\text { Urinary tract infection }}$

$\underline{30} \quad \underline{(12)}$

$\underline{\text { Gastrointestinal or biliary tract infection }}$

$\underline{23} \underline{(9)}$

$\underline{\text { Catheter-related infection }}$

$\underline{15} \underline{(6)}$ 


\section{(Continued on next page)}

$\underline{\text { Table 1. (Continued) }}$

Characteristics $^{1}$

$\underline{\text { Skin or soft tissue infection }}$

$\underline{11} \underline{(4)}$

$\underline{\text { Endocarditis }}$

$\underline{9} \quad \underline{(3)}$

$\underline{\text { Bone or joint infection }}$

$\underline{8} \quad \underline{(3)}$

$\underline{\text { Neutropenic patient with unexplained fever }}$

$\underline{7} \quad \underline{(3)}$

$\underline{\text { Other infections }}$

$\underline{22} \underline{(8)}$

$\underline{\text { Other diagnoses }}$

$\underline{79} \quad \underline{(30)}$

Length of stay, median (IQR), $d$

$\underline{12} \underline{(5-22)}$

Abbreviations: IQR, interquartile range (i.e., $25^{\text {th }}$ and $75^{\text {th }}$ percentiles).

${ }^{1}$ Values were missing for temperature (no. $=38$ ) and intravenous access device (no. $=9$ ).

${ }^{2}$ Immune suppression included CD4 lymphocyte cell count $<250 / \mathrm{mm}^{3}$, neutrophil cell count $<500 / \mathrm{mm}^{3}$, active cancer, transplantation, corticosteroid therapy, and immunosuppressive therapy.

${ }^{3}$ One true-positive case also had a contaminated blood culture. 
Table 2. Microorganisms isolated from positive blood cultures obtained from patients with bloodstream infection.

$\underline{\text { Microorganism }^{1}} \quad \underline{\text { No. isolates }}$

$\underline{\text { Enterobacteriaceae }} \underline{10}$

Staphylococcus aureus $\quad \underline{9}$

$\underline{\text { Coagulase-negative staphylococci }} \quad \underline{9}$

Streptococcus spp. $\quad \underline{4}$

Enterococcus spp. $\quad \underline{1}$

$\underline{\text { Pseudomonas aeruginosa }} \quad \underline{1}$

${ }^{1}$ Staphylococcus aureus and Enterococcus spp. were isolated from the same bottle in one patient with polymicrobial bloodstream infection. 
Table 3. Appropriateness of blood cultures and antimicrobial therapy.

Characteristics

No./total no. $\quad$ (percentage $[95 \%$

$\underline{\mathrm{CI}})$

Very/fairly appropriate indication for blood culture

$\underline{\text { bacterial (aerobic and anaerobic) }}$

$\underline{238 / 260} \underline{(91[87-95])}$

fungal

$\underline{77 / 103} \underline{\underline{(75[65-83])}}$

$\underline{\text { Number of bacterial blood cultures }}$

$\underline{1}$

$\underline{72 / 260} \quad \underline{(28[22-33])}$

$\underline{2-4}$

$\underline{118 / 260} \underline{(45[39-52])}$

$\geq 5$

$\underline{70 / 260} \quad \underline{(27[22-33])}$

$\underline{\text { Interval between consecutive blood cultures }<24 \mathrm{~h}^{1}}$

$\underline{135 / 188} \underline{(72[65-78])}$

$\underline{\text { Site of blood culture }}$

very/fairly appropriate

$\underline{228 / 260} \underline{(88[83-91])}$

very/fairly inappropriate

$\underline{15 / 260} \underline{(6[3-9])}$

$\underline{\text { unspecified }}$

$\underline{17 / 260} \quad \underline{(6[4-10])}$

$\underline{\text { Volume of blood per bottle for adult patients }}{ }^{2}$

$\geqq 10 \mathrm{~mL}$

$\underline{121 / 919} \underline{(13[11-15])}$

$\underline{8-9 \mathrm{~mL}}$

$\underline{201 / 919} \underline{(22[19-25])}$

$\leq 8 \mathrm{~mL}$

$\underline{597 / 919} \underline{(65[62-68])}$

$\underline{\text { Volume of blood per bottle for pediatric patients }}{ }^{2}$

$\geqq 3 \mathrm{~mL}$

$\underline{29 / 36} \quad \underline{(80[64-92])}$

$\underline{1-3 \mathrm{~mL}}$

$\underline{2 / 36} \underline{(6[1-19])}$

$\leq 1 \mathrm{~mL}$

$\underline{5 / 36} \underline{(14[5-29])}$ 
$\underline{\text { Table 3. (Continued) }}$

$\underline{\text { Characteristics }}$

No./no. total (percentage [95\%

$\underline{\mathrm{CI}])}$

Empirical antimicrobial therapy for patients with true-

positive or contaminated blood cultures

very/fairly appropriate

$\underline{23 / 38} \underline{(60[43-76])}$

very/fairly inappropriate

$\underline{5 / 38} \quad \underline{(13[4-28])}$

$\underline{\text { not used }}$

$\underline{10 / 38} \underline{(26[13-43])}$

Adjustment of antimicrobial therapy after the results of true-

positive or contaminated blood cultures were available

very/fairly appropriate

$\underline{27 / 38} \underline{(71[54-85])}$

Abbreviations: CI, confidence interval

${ }^{1}$ Seventy-two patients with only one blood culture were omitted from the analysis of the interval between consecutive blood cultures.

${ }^{2}$ Overall, 919 and 36 bottles were obtained from 224 adult and 36 pediatric patients, respectively. 
Figure 1. Rank of positivity of the first 11 sets of aerobic and anaerobic blood cultures. $\underline{\text { One true-positive case also had a contaminated blood culture. }}$ 


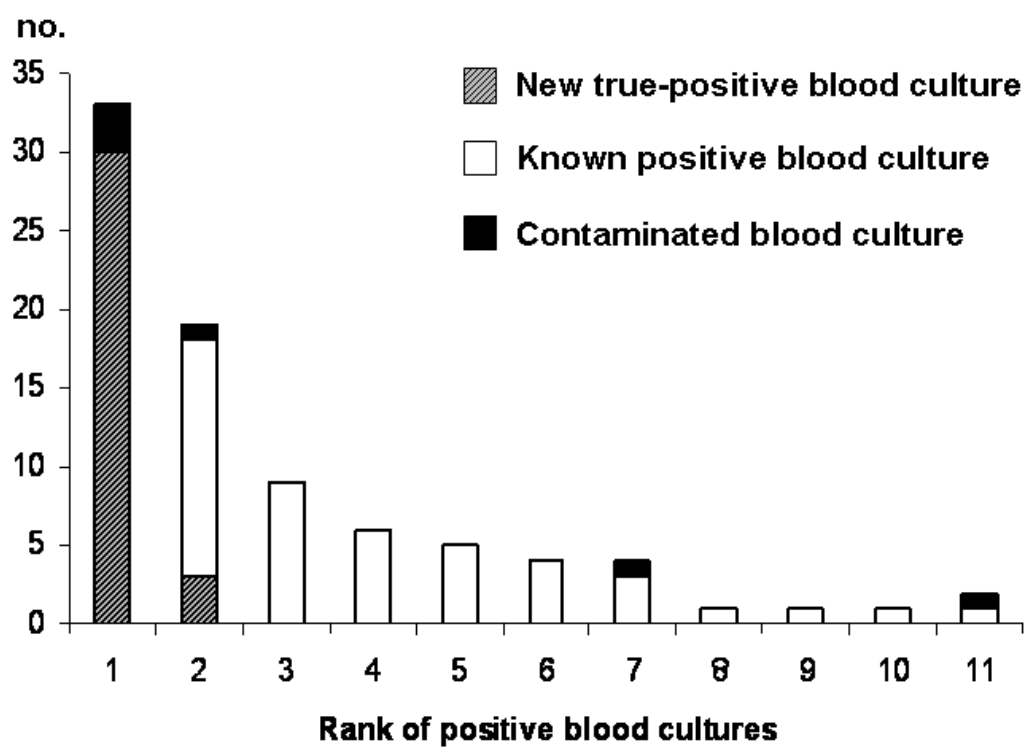

